\title{
Anomalous burning rates of flamelets induced by self-similar multiple scale (fractal and spiral) initial fields
}

\author{
Nadeem A. Malik ${ }^{1,2, *}$ and J. C. H. Fung ${ }^{2}$ \\ ${ }^{1}$ Department of Mechanical Engineering, Imperial College of Science, Technology, and Medicine, Exhibition Road, \\ London SW7 2BX, England \\ ${ }^{2}$ Department of Mathematics, Hong Kong University of Science and Technology, Clear Water Bay, Hong Kong
}

(Received 31 March 2000)

\begin{abstract}
In contrast to the classical problem of a single idealized flamelet (which is described by a nonlinear reaction-diffusion equation of motion) which propagates at a constant burning rate, self-similar multiple scale fields, whether fractal or nonfractal, induce anomalous rates of burning determined by the space-filling properties of the initial field. We compare the regimes induced by (line-cuts through) three specific geometries with distinct space-filling characteristics: (1) an algebraic spiral which has capacity (box-counting dimension) $D_{k}$ $>0$, and fractal dimension $H=0 ;(2)$ an exponential spiral which has $D_{k}=0$ and $H=0$, and geometric ratio $R>1$; (3) a fractal Cantor dust which has $D_{k}=H>0$. The (nondimensional) burning rate $U_{B}$ induced by all three geometries takes the general form $U_{B} \sim \mathcal{F}\left(\tau^{-\zeta}\right)$, where $\mathcal{F}$ is a function whose form depends on the specific geometry, $\zeta$ is an exponent that contains the space-filling characteristic of the geometry, and $\tau$ is a nondimensional time. (1) For the algebraic spiral, $\mathcal{F}(x)=1(x)$, and $\zeta=D_{k} ; \mathcal{F}$ is continuous. (2) For the exponential spiral, $\mathcal{F}(x)=\ln (x)$, and $\zeta=1 /(R-1) ; \mathcal{F}$ is continuous. (3) For the fractal Cantor dust, $\mathcal{F}^{u}(x)$ $=1(x)$, and $\zeta=H$ (for the envelope); $\mathcal{F}$ itself is a step-like discontinuous function. Thus, as $D_{k} \rightarrow 0$, or as $H \rightarrow 0$, or as $R \rightarrow \infty$, then $\zeta \rightarrow 0$ and $U_{B} \rightarrow$ const; and as $D_{k} \rightarrow 1$, or as $H \rightarrow 1$, (space filling) then $\zeta \rightarrow 1$; and as $R \rightarrow 1$ (space filling) then $\zeta \rightarrow \infty$. Two numerical methods, a fundamental (Eulerian) solution to the equation of motion and a Lagrangian model for flamelet propagation, confirm these theoretical predictions. The Lagrangian model is based on the idealized flamelet as a "point" with finite flame thickness $\Delta_{L}$, (which is determined by the two-flamelet collision process), propagating with a given flame speed $U_{L}$. The Lagrangian model allows simulations in parameter ranges not easily accessible by the fundamental method (such as the case for the fractal Cantor dust). Interestingly, the linear regime of scalar diffusion in an algebraic spiral field displays the same dependence on $D_{k}$ as in the present reaction-diffusion case. The nonlinear regime of advection-diffusion (Burger turbulence) shows a different dependence on $D_{k}$.

PACS number(s): 47.53.+n, 68.35.-p, 94.10.Lf, 82.40.Fp
\end{abstract}

\section{INTRODUCTION}

Much interest has been excited in the scientific community in recent years on the impact of self-similar multiple scale fields, in the initial and/or boundary conditions or where such geometries are generated within the fluid flow itself, on physical processes. Many classical problems which have standard solutions show dramatically different physical behavior due to these types of complex initial and/or boundary conditions.

Gurbatov and Crighton [1] investigated the anomalous decay of energy of fractal signals in the context of onedimensional "Burgers turbulence" which is described by solutions of the nonlinear Burgers' equation. They showed that the energy decay law for a fractal signal, consisting of an idealized initial field of pulses, was determined by its capacity $D_{k}$. These ideas were further developed by Angillela and Vassilicos [2] who looked at the idealized one-dimensional sequences of both alternating and nonalternating pulses in fractal and spiral fields. Vassilicos [3] also looked at the decay of scalar fields subjected to molecular diffusion in fractal or spiral initial fields. These regimes are described by

\footnotetext{
*Corresponding author. Email address: N.A.Malik@ic.ac.uk
}

the linear diffusion equation subject to the above initial conditions. His findings were similar to those of Gurbatov and Crighton.

Other researches in this field include Berry [4], She et al. [5], van der Berg [6], and Fleckinger et al. [7]. See also Ref. [8] for a general overview of the physics and biology of fractals.

In this paper, we focus our attention on the impact that different types of one-dimensional self-similar multiple scale fields have on the burning regime of groups of flamelets. The geometries of such fields is characterized by capacities (or box-counting dimensions) $D_{k}$, by fractal dimensions $H$, and by geometric ratios $R$. In contrast to the linear diffusion regimes investigated in [3], the present context of flamelets is described by a set of strongly nonlinear reaction-diffusion partial differential equations with fractal or spiral initial field configurations. A key question is, how is the burning rate determined by $D_{k}, H$, or $R$ in the different fractal and spiral fields? What are the similarities, if any, and the differences with other physical systems, such as in [1] and [3]?

Flames, broadly speaking, are exothermic chemical reactions with a burning rate which is a strongly increasing function of the temperature $T$. In many situations, there is a strong nonlinear coupling between the chemical reactions, 
the heat released and the underlying fluid flow which is often turbulent. A theory of chemical burning in fluid flows is far from complete, but there are situations where the burning regime can be simplified to the point of being analytically and/or numerically tractable. The simplest such case is that of the flamelet regime; formally it is described by a system of nonlinear reaction-diffusion equations in the species and temperature variables. This system has been completely solved for asymptotically large activation energies. This is essentially due to the fact that, in the limit of high activation energy, the nonlinear reaction source term is localized within a zone which is thin compared to the total flame thickness; this yields a unique and constant flame speed $U_{L}$ which is directly proportional to the burning rate. See Williams [9].

It is of some interest here to contrast the physical regimes of flamelets, which is the context of this paper, and that of the Burgers 'turbulence' which is the context of [1] and [2], and also the linear scalar diffusion decay regime of [3]. The first is described by a set of reaction-diffusion equations [see Eqs. (2) and (3)], while the second is described by advectiondiffusion equations. Both regimes are highly nonlinear. But whereas in the Burgers turbulence the diffusion is acting constantly leading to an essentially unsteady decaying solution with increasing correlation length scale, in the reactiondiffusion system of flamelets the diffusion is balanced by the reaction kinetics thus leading to an essentially steady propagating solution with constant laminar flame speed. Part of the interest in this paper is to investigate the differences and similarities in the way fractal/spiral initial conditions determine the anomalous regimes in various physical contexts.

The rest of the paper is organized as follows. In Sec. II, we review the theory for a single flamelet, and in Sec. III we pose the problem of the burning rate for groups of flamelets in self-similar multiple scale initial conditions. In Sec. IV, we look at the two-flamelet collision process which gives interaction time scales and a measure of the laminar flame thickness. In Sec. V, we present theories for the burning rates, first for the case of ideal zero-thickness flamelets and then for finite-thickness flamelets. In Sec. VI, we develop a Lagrangian based flamepoint model for flamelet propagation. Numerical solutions of the fundamental partial differential equation and the results from the flamepoint model are presented and compared to the analytical solutions in Sec. VII. We conclude with a summary of the results and some comments in Sec. VIII.

\section{SINGLE FLAMELETS}

In the flamelet regime, the combustion is simplified to the case of the single step pre-mixed burning of a mixture of fuel $(F)$ and oxidizing $(O)$ gases which produce product $(P)$ gas and a quantity of heat $(Q)$, viz

$$
\nu_{F} F+\nu_{O} O \rightarrow P+Q,
$$

where $\nu_{F}$ and $\nu_{O}$ are the stoichiometric coefficients of the reaction. The flame propagates with subsonic speed by the diffusive transport of mass and energy. The chemical kinetics is assumed to be governed by the nonlinear Arhenius' law with a chemical time scale $\tau_{r}$, (see Ref. [10]). We define $\theta=T-T_{U} / T_{B}-T_{U}, 0 \leqslant \theta \leqslant 1$, as the reduced temperature, where $T_{U}$ and $T_{B}$ are the unburnt and burnt temperatures, respectively. (Henceforth, the subscript $U$ will refer to quantities in the unburnt mixture, while $B$ will refer to quantities in the burnt mixture.) The reduced chemical mass fraction is $\chi=Y / Y_{U}$ where $Y$ is the species mass fraction, and $0 \leqslant \chi$ $\leqslant 1$. If $E$ is the activation energy then the nondimensional activation energy is defined by $Z=E\left(T_{B}-T_{U}\right) / R_{C} T_{B}^{2}$, also known as the Zeldovich number. $R_{c}$ is the perfect gas constant.

The thermal diffusivity is defined by $D_{\theta}=\lambda / \rho C_{p}$, where $\lambda$ is the thermal conductivity, $\rho$ is the density, and $C_{p}$ is the specific heat. The mass diffusivity is $D_{m}$, and the Lewis number is $L e=D_{\theta} / D_{m}$. Then we are left with two coupled nonlinear reaction-diffusion partial differential equations in $\chi$ and $\theta$, viz

$$
\begin{gathered}
\rho \frac{\partial \chi}{\partial t}-\frac{\partial}{\partial x}\left(\rho D_{m} \frac{\partial \chi}{\partial x}\right)=-\rho W_{r} \\
\rho \frac{\partial \theta}{\partial t}-\frac{\partial}{\partial x}\left(\rho D_{\theta} \frac{\partial \theta}{\partial x}\right)=\rho W_{r}
\end{gathered}
$$

with identical boundary conditions for $\theta$ and $1-\chi$,

$$
\theta=1-\chi=0 \text { at } x=-\infty ; \quad \text { and } \theta=1-\chi=1 \text { at } x=\infty .
$$

$W_{r}=A / \tau_{r} w_{r}(\theta, Z)$ is the reaction rate at temperature $\theta$ $=\theta(x, t)$, and $\tau_{r}=\tau_{r}\left(T_{B}\right)$ is the chemical time scale taken at the burnt temperature. $A$ is a constant which we take to be equal to unity in all of our simulations. (For convenience, henceforth we will suppress all parameters except $\theta$ in $W_{r}$.)

In the special case where the Lewis number is unity, Le $=1$, and if we also neglect gas expansion, $\rho_{B}=\rho_{U}=\rho=1$, the equations themselves are identical with identical boundary conditions, and the problem is reduced to solving just one nonlinear thermal reaction-diffusion equation in $\theta$,

$$
\frac{\partial \theta}{\partial t}-D_{\theta} \frac{\partial^{2} \theta}{\partial x^{2}}=\frac{1}{\tau_{r}} w_{r}(\theta)
$$

with the same boundary conditions.

The total consumed mass $M(t)$ at time $t$ in the domain is

$$
M(t)=\int_{-\infty}^{+\infty} \rho \theta(x, t) d x,
$$

and the burning rate (rate of mass consumption) $\rho U_{B}(t)$ is given by

$$
\rho U_{B}(t)=\frac{d M(t)}{d t}
$$

In general, Eq. (4) cannot be solved analytically because of the strongly nonlinear source term $w_{r}(\theta)$ on the right hand side. However, it has been shown that in the limit where the reduced activation energy $Z \rightarrow \infty$ then $W_{r}(\theta)$ becomes increasingly localized at $\theta=1$; this yields a stiff equation in which heat diffuses within an extremely localized neighborhood just behind the flame front, of thickness $\Delta_{L}(Z)$. The released energy diffuses and preheats the unburnt gases just in front where the combustion is ignited. 


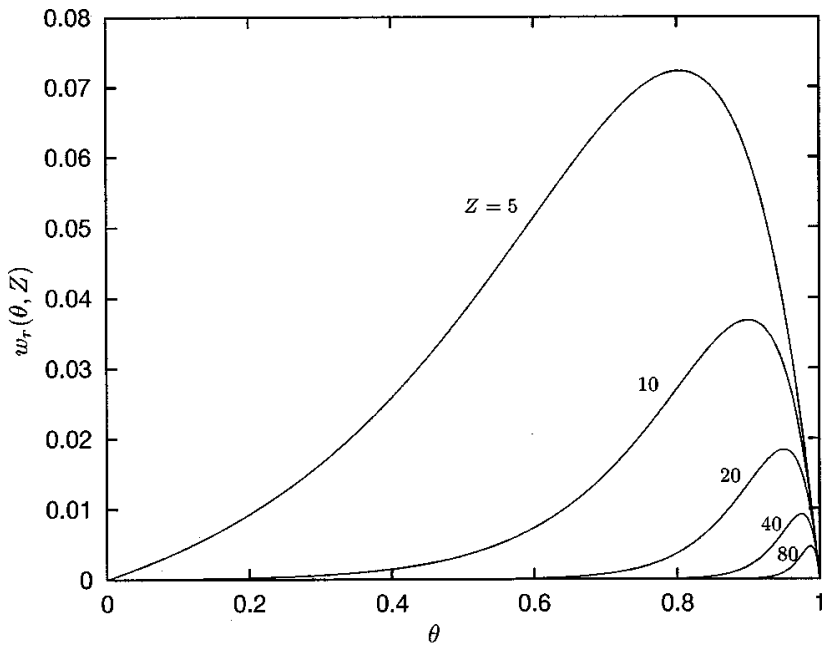

FIG. 1. A sequence of reaction rate profiles, $w_{r}(\theta, Z)$ against $\theta$ the reduced temperature, for $m=1$ for different Zeldovich number (nondimensional activation energy) $Z$.

The nonlinear term $w_{r}$ in Eq. (4) must take a particular form; for an $m^{\text {th }}$ order chemical reaction, a typical reaction rate profile for $w_{r}$ is,

$$
w_{r}(\theta)=(1-\theta)^{m}\{\exp (-Z(1-\theta))-\exp (-Z)\} .
$$

A sequence of $w_{r}$ curves as $Z \rightarrow \infty$ is shown in Fig. 1 for $m=1$. The curves become localized as $Z \rightarrow \infty$ for all $m$, with the peak occurring at $\theta_{\max } \approx 1-m / Z \rightarrow 1$; the peak value at $\theta_{\text {max }}$ is $w_{r} \approx(m / Z)^{m} \rightarrow 0$ as $Z \rightarrow \infty$. The region where most of the activity occurs is a very thin region centered around the peak at $\theta_{\max }$ of extent $\Delta \theta \sim O(1 / Z)$; thus the reaction zone thickness scales as $\delta_{r} \sim O\left(\Delta_{L} / Z\right)$. The flame front advances like a plane wave with a constant and unique flame speed $U_{L}$ which is equal to the rate of burning $U_{B}$. The flamelet structure is steady in the frame of reference moving with speed $U_{L}$ at the flame front. Across this region, the reduced temperature goes from $\theta=0$ (unburnt) to $\theta=1$ (burnt). Figure 2 shows the flame structure.

Using (7) for the reaction term, the exact result for $Z$ $\rightarrow \infty$ is (Clavin [10]):

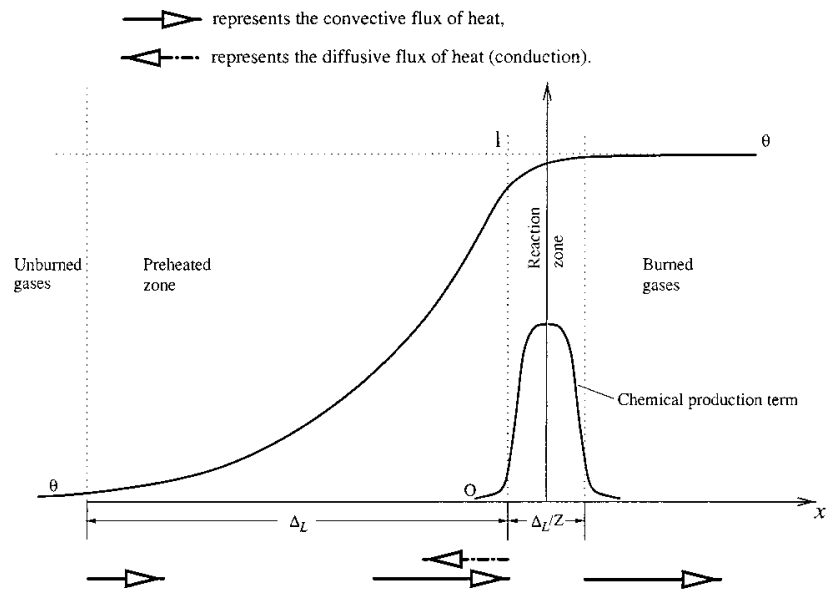

FIG. 2. A sketch of the flamelet structure. $\Delta_{L}$ is the flame thickness.

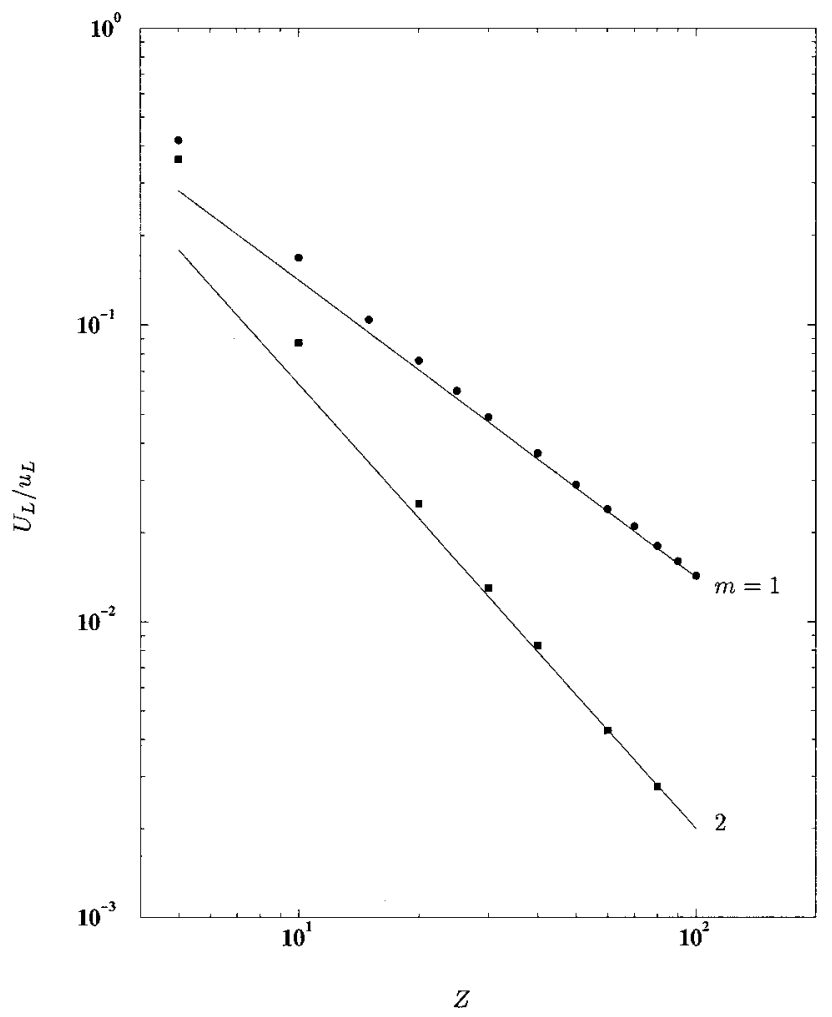

FIG. 3. Log-log plot of the laminar flame speed, $U_{L} / u_{L}$ against $Z ; u_{L}=\sqrt{D_{\theta} / \tau_{r}} ; D_{\theta}$ is the thermal diffusivity, $\tau_{r}$ is the chemical time scale.), see Eq. (8). Solid lines are the asymptotic solutions for $Z \rightarrow \infty$ for different $m$ (the order of the kinetic reaction). The symbols are our computed solutions of Eq. (4): $m=1$ (circles), $m=2$ (boxes).

$$
\frac{U_{L}}{u_{L}}=\sqrt{\frac{2 \Gamma(m+1)}{Z^{m+1}}},
$$

where $u_{L}=\sqrt{D_{\theta} / \tau_{r}}$, and $\Gamma(m)$ is the Gamma function of order $m$. Figure 3 shows the plot of $U_{L} / u_{L}$ against $Z$ for different orders $m=1,2$ (solid lines) according to the above law.

In the rest of the paper, unless otherwise stated, all times, velocities and lengths are nondimensionalized by, respectively, $\tau_{r}, u_{L}$ and $\delta_{L}=\sqrt{D_{\theta} \tau_{r}}$; except in figures where we show the entire quantities that are plotted.

\section{POSING THE PROBLEM OF GROUPS OF FLAMELETS}

Suppose that instead of a single flamelet, we initiate at time $t=0$ a group of flamelet-pairs centered at positions $\left\{x_{n}\right\}, n=1, \ldots, N$ within a given domain of interest. A flamelet-pair consists of two flamelets arranged back-to-back so that the two flamelets propagate in opposite directions. At certain times after release, neighboring flamelets will collide and interact and the volume of gas between them will be consumed; the two colliding flamelets will then vanish from the domain. The total number of flamelet-pairs at time $t$, $N(t)$ say, in the domain will diminish by 1 , viz $N \rightarrow N-1$, just after a collision. As time advances, $N \rightarrow 0$.

At a given time $t$ after release the $2 N(t)$ flamelets can be grouped into two sets; those that are not interacting with any 


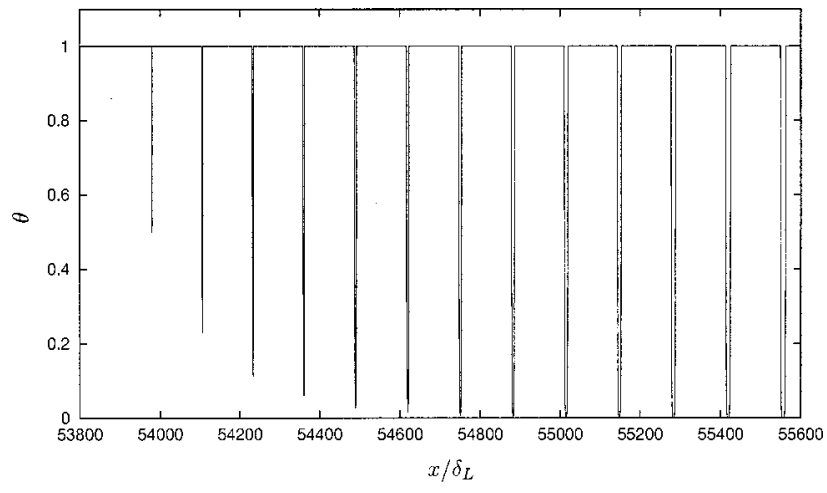

FIG. 4. The $\theta$-profile against the distance $x / \delta_{L}$ at the leading edge of a group of flamelets from a numerical simulation in which flamelet-pairs were released in an algebraic spiral-cut configuration. Note how the successive flamelet collisions are at different phases of the interaction process. $\left(\delta_{L}=\sqrt{D_{\theta} \tau_{r}}\right.$.

other flamelet and as such can be treated individually as single flamelets - there are $2 N_{s}(t)$ such single flamelets at time $t$. Each one of these flamelets is localized within a subdomain $\square^{n}$ and centered at a point $x_{n}(t) \in \square^{n}$ for $n$ $=1, \ldots 2 N_{s} ; \square^{n} \cap \square^{n^{\prime}}=\varnothing$ for $n \neq n^{\prime}$. The remaining $2 N_{i}$ $=2\left(N-N_{s}\right)$ flamelets are in the process of collision (pairwise) and they form the second group of flamelets. This group must be treated as a whole. Figure 4 shows the leading edge of the $\theta$-profile from a numerical simulation at some time after release. The leading-edge is where flamelets are at varying phases in the collision process. In this case there are about $N_{i}=10$ such collision processes.

We seek an approximation to the burning rate at time $t$ $>0$ after release. From Eqs. (5) and (6), with $\rho=1$,

$$
\begin{aligned}
U_{B}(t) & =\frac{d}{d t}\left\{\int_{-\infty}^{\infty} \theta(x, t) d x\right\}=\sum_{n=1}^{2 N_{s}} \frac{d}{d t}\left\{\int_{\square^{n}} \theta(x, t) d x\right\}+I(t) \\
& =2 N_{s}(t) U_{L}+I(t),
\end{aligned}
$$

where the sum is over all the $2 N_{s}(t)$ noninteracting flamelets taken individually at time $t$; the contributions from the $2 N_{i}$ interacting flamelets is contained in $I(t)$. (In the integral, the subdomains $\square^{n}$ must be interpreted in a Lagrangian sense since these subdomains are located where the flamelets are at time $t$.)

If $I \ll 2 N_{s}(t) U_{L}$ then this term can be neglected; we can expect this to happen when $N(t) \approx N_{s}(t) \gg N_{i}$. Then, the burning rate is given approximately by

$$
U_{B}(t) \approx 2 N(t) U_{L}, \quad N(t) \gg N_{i} .
$$

Equation (10) is a general approximation for an arbitrary initial set of release points $\left\{x_{n}\right\}$. Suppose now that the set of release points $\left\{x_{n}\right\}$ has a specific geometric configuration can an analytical expression for the burning rate $U_{B}(t)$ be obtained? In this paper, we consider three contrasting geometries:

(1) An algebraic spiral of the form (in polar coordinates) $r \sim(\phi / 2 \pi)^{-p}$ for $\phi>0, p>0$;

(2) An exponential spiral (in polar coordinates) $r$ $\sim \exp (a \phi / 2 \pi)$ for $\phi>0, a>0$;

(3) A Fractal Cantor Dust on the real line. (a)

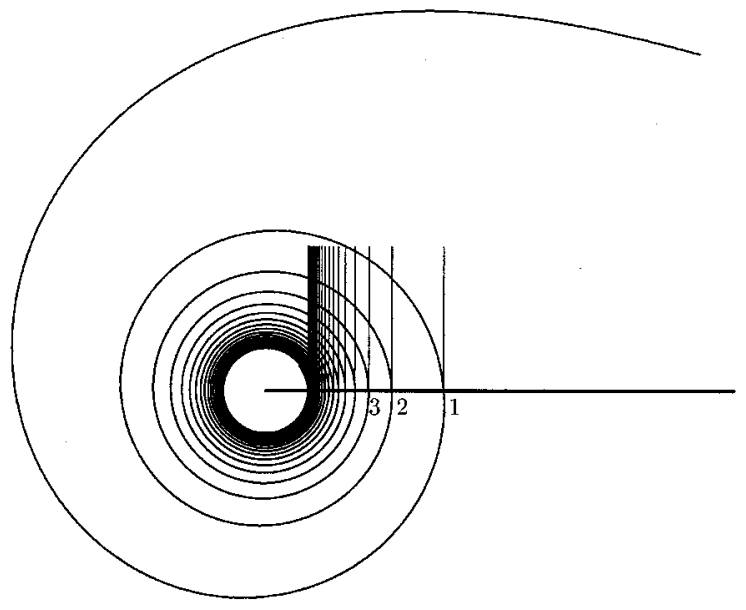

(b)

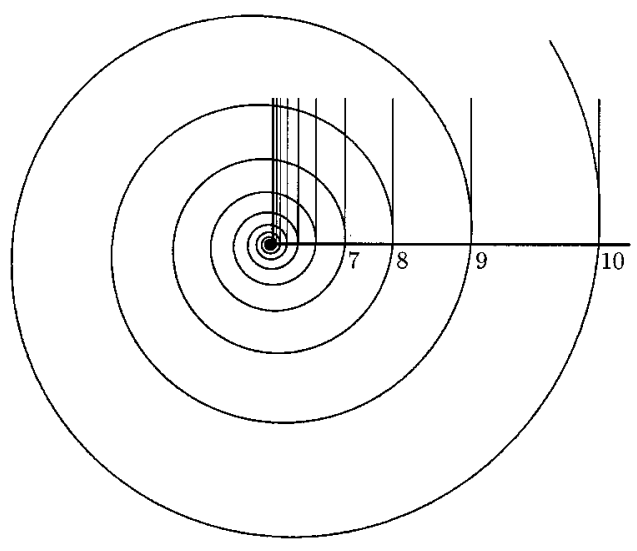

(c)

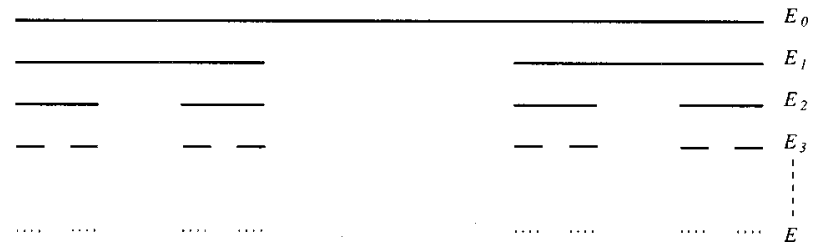

FIG. 5. The geometries that are examined in this paper. The distribution of flamelet-pairs $\left\{x_{n}\right\}$ are where the $\mathrm{x}$-axis cuts the geometrical objects (shown as vertical lines in (a) and (b)). Refering to Sec. III: (a) The algebraic spiral, (in polar coordinates) $r$ $\sim(\phi / 2 \pi)^{-p}$; here $p=0.5$. (b) The exponential spiral, $r$ $\sim \exp (a \phi / 2 \pi)$; here $a=0.5$. Refering to Sec. V 3: (c) The construction of the middle third Cantor dust, with $R=1 / 3, M=2$, and thus the fractal dimension $H=S=\ln (M) / \ln (1 / r)=0.63093$.

While all three geometries are multiple scaled and selfsimilar, they also possess distinct space-filling characteristics. The algebraic spiral, Fig. 5(a), is characterized by a nontrivial capacity, or box-counting dimension, $D_{k}>1$ and a trivial fractal dimension $H=0$; it is a smooth nonfractal object which is locally self-similar; it is singular in the sense that a point moving along the spiral arm approaches the center in ever tighter fashion. The exponential spiral, Fig. 5(b), has the trivial $D_{k}=1$ and $H=0$; its geometry is characterized by the geometric ratio $R$ which defines the ratio of successive 
points along a line-cut through its center. It is also locally self-similar but with a milder singularity than the exponential spiral. The fractal Cantor Dust, Fig. 5(c), has $D_{k}>0$ and $H$ $>0$. A fractal object is characterized by a global selfsimilarity in the sense that such objects possess fine structure and detail on arbitrarily small scales at all points - this global self-similarity is perhaps approximate, or even statistical. Mathematically they are said to have a nontrivial Hausdorff-Besicovich (fractal) dimension $H>0$ which appears as the exponent in the scaling law for such an object, viz an $H$-dimensional Hausdorff measure $\mathcal{H}(F)$ on some set $F \in R^{n}$ scales as $\mathcal{H}(\lambda F)=\lambda^{H} \mathcal{H}(F)$ where $\lambda>0$ is a real number. Box-counting dimensions $D_{k}$, and fractal dimensions, $H$ are indicative of the space-fillingness of curves and geometrical objects; the reader is referred to Falconer [11] for details. However, it should be noted here that $D_{k}$ and $H$ are not exclusive means of defining the space-filling properties of curves. For instance, in the example of the exponential spiral above, clearly as $a \rightarrow 0$, then the spiral curve tends to become space filling even though both $D_{k}=0$ and $H$ $=0$.

The inclusion of the exponential spiral in our set of geometrical objects is of particular interest since it has not been considered in the previous works ( [1-3]). Although it is a smooth nonfractal curve, it is nevertheless multiple scaled, self-similar and contains a singularity and it can also be space filling in the sense described above. It offers a type of geometry to investigate and it is important to see whether a nonfractal $\left(D_{k}=0, H=0\right)$ field can also induce anomalous physical regimes.

In fact, we will be dealing with the set of points where a line (the $\mathrm{x}$-axis) cuts the spiral objects through its center. The geometrical properties of these sets of points are similar to the original geometrical objects in the sense that the boxcounting dimension of the points on the spiral-cut is $D_{k}^{\prime}$ $=D_{k}-1$. (Line cuts which are off the center possess similar properties except that the range of scales over which the space-filling properties are apparent is reduced.)

Before we look at the general theory for groups of flamelets, we first look at the two-flamelet collision process.

\section{THE TWO-FLAMELET COLLISION PROCESS}

\section{A. The interaction time scale $T_{i}$}

When two flamelets are released at some distance apart, initially they move towards each with total speed $2 U_{L}$. Some time later, the flamelets interact and consume all the unburnt gas between them $(\theta=1)$ at which point the two flamelets vanish from the domain. The interaction of flamelets is an unsteady nonlinear process, and a full treatment would include physical effects such as gas expansion, instability, thermal radiation and long-range interactions. However, within the framework of idealized flamelets, we will ignore these effects.

Stiff nonlinear systems possess widely differing length scales; here, the laminar flame thickness $\Delta_{L}$ is the inner length scale and $L$, the scale of the domain, is the outer length scale. We have computed the solution of the PDE (4) in a one-dimensional domain using a Crank-Nicholson finite difference scheme with, typically, a density of $2^{23}$ grid points per unit length which is adequate for all of our computations.

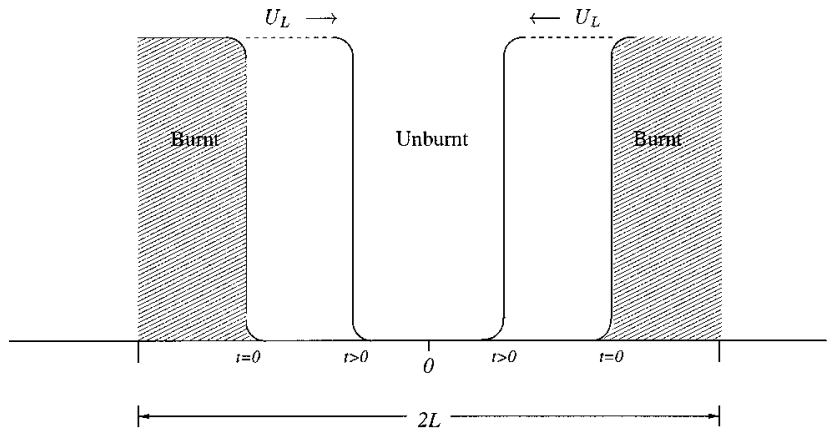

FIG. 6. The two-flamelet collision process: a sketch of the $\theta$-profiles at the initial time $t=0$ and at some time $t>0$ before interaction has began. The two flamelets approach each other with total speed of $2 U_{L}$ before they interact in any way. $2 L$ is the length of the domain considered.

With an outer length scale of $L=1$, this means that the total number of points in a simulation is of the order of $2^{23}$. Two flamelets propagating towards each other are initiated as step-functions in $\theta$ in a domain $[-L, L], L>0: \theta(x)=1$ for $-L \leqslant x \leqslant-L+l_{0}$ and $L-l_{0} \leqslant x \leqslant L$, for some $0<l_{0} \ll L$; and $\theta(x)=0$ otherwise. In other words, the two flamelets move in from the edges of the domain towards the center, see Fig. 6 . There is a short transition period during which the flame fronts adjust to their smooth quasisteady profile; the initial distance between the flamelets $2\left(L-l_{0}\right) \gg 2 \Delta_{L}$ so that they have sufficient time to attain to their steady profile before they interact in any way.

In Fig. 3 we also show the results from our simulations for $m=1$ (diamonds) and 2 (crosses) for various $Z$. The laminar flame speed $U_{L} / u_{L}$ decreases with both $m$ and $Z$. The computed results approach the asymptotic law in Eq. (8) as $Z \rightarrow \infty$; this validates our numerical scheme.

Figure 7 shows a typical time sequence of the $\theta$-profiles of colliding flamelets as the enclosed volume is consumed; this particular case is for $m=1$ and $Z=40$.

Figure 8 shows plots of the burning rate $U_{B} / u_{L}$ against time $t / \tau_{r}$ for $m=1$, and $Z=20,40$ and 80 . The flamelets move towards each other with constant speed $2 U_{L}$ for some time; later, the flamelets interact leading to an accelerated rate of burning which peaks sharply before falling almost immediately thereafter to zero as the enclosed volume is totally consumed. Note that although $U_{B}$ peaks sharply it re-

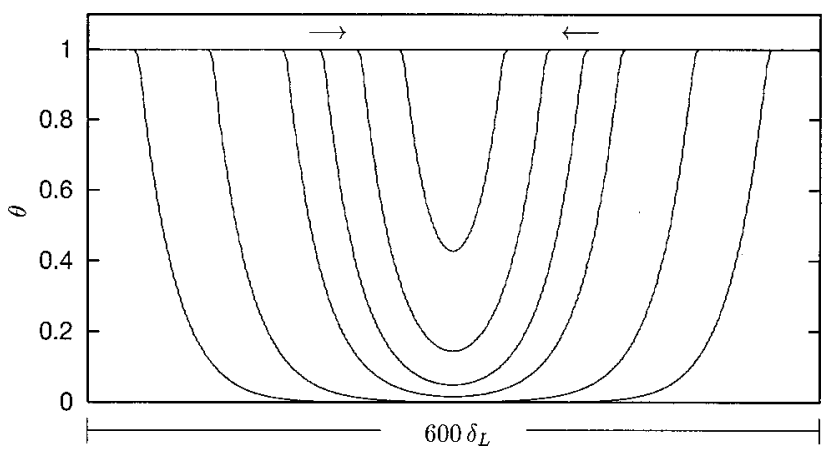

FIG. 7. The two-flamelet collision process: computed $\theta$-profiles at different times during the two-flamelet collision process. Here, we have taken $m=1$ and $Z=40$. Other cases show similar qualitative behavior. 


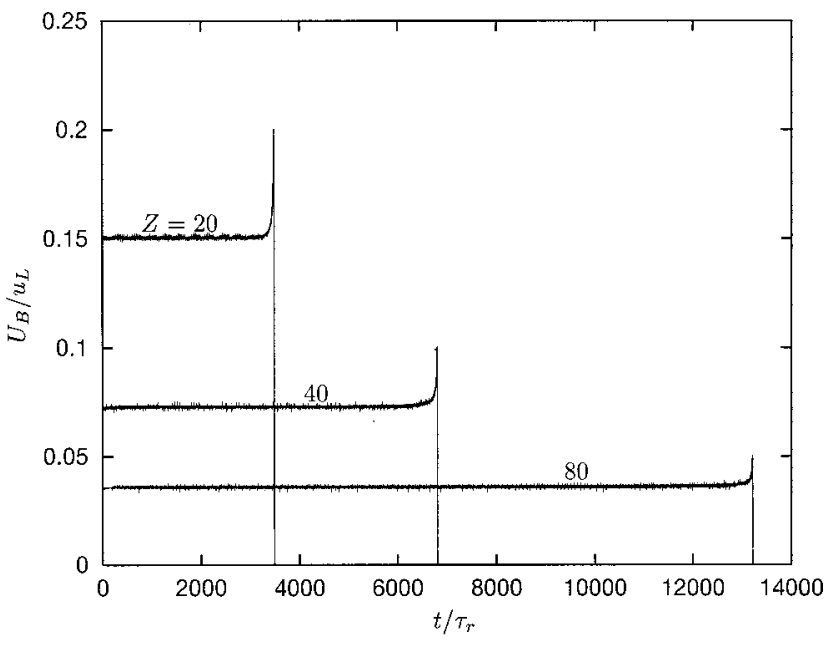

FIG. 8. The two-flamelet collision process: the computed burning rates $U_{B}(t) / u_{L}$ against the time $t / \tau_{r}$ for three cases with $m=1 ; Z=20,40$, and 80 .

mains of the same order, $O\left(U_{L}\right)$, even at the peak value.

Let the time over which this interaction takes place be $T_{i}$. The time taken for a flamelet to move a distance equal to one flame thickness $\Delta_{L}$ is $T_{L}=\Delta_{L} / U_{L}$. Then $T_{i} / T_{L}$ $=T_{i} U_{L} / \Delta_{L}$. The results show that $T_{i} / T_{L} \ll 1$, i.e., the collision of the two oncoming flamelets occurs on a very short time scale compared to the time it would take if there were no interaction, viz

$$
\frac{T_{i}}{T_{L}} \approx 0 .
$$

It is as if the flamelets interact and vanish almost instantaneously upon first impact.

\section{B. The flame thickness, $\Delta_{L}$}

Models of flame propagation encounter the problem of defining a precise value for the flame thickness $\Delta_{L}$. Typically, an ad hoc value for $\Delta_{L}$ is taken to be the distance between where $\theta=0.05$ and 0.95 , i.e., within $5 \%$ of the extreme $\theta$-values. But for our theory such a definition is not precise enough to be useful since there is no reason why $5 \%$ should be any better than $0.1 \%, 1 \%$ or $6 \%$, etc. What we need is a more physically based definition of the flame thickness that is both precise and unique.

The essential physical phenomena in flamelet propagation is the process of mass consumption, in the manner described in Sec. II. In the two-flamelet collision process, as we have just seen, two flamelets consume the mass enclosed between them in a time that can be computed precisely. Suppose that we could model flamelet propagation in a Lagrangian frame as the motion of a "point" with finite thickness $\Delta_{L}$ propagating with given velocity $U_{L}-$ this is an idea which we will use in Sec. VII in the flamepoint model. Then we can define a flame thickness $\Delta_{L}$ as that value of $\Delta_{L}$ when the time taken for the two-flamelet collision process to completely consume the enclosed mass as computed from the flamepoint model (Sec. VII) is identical to the time for the same process as computed from the fundamental solution of Eq. (4). $\Delta_{L}$ is an input parameter in the flamepoint model

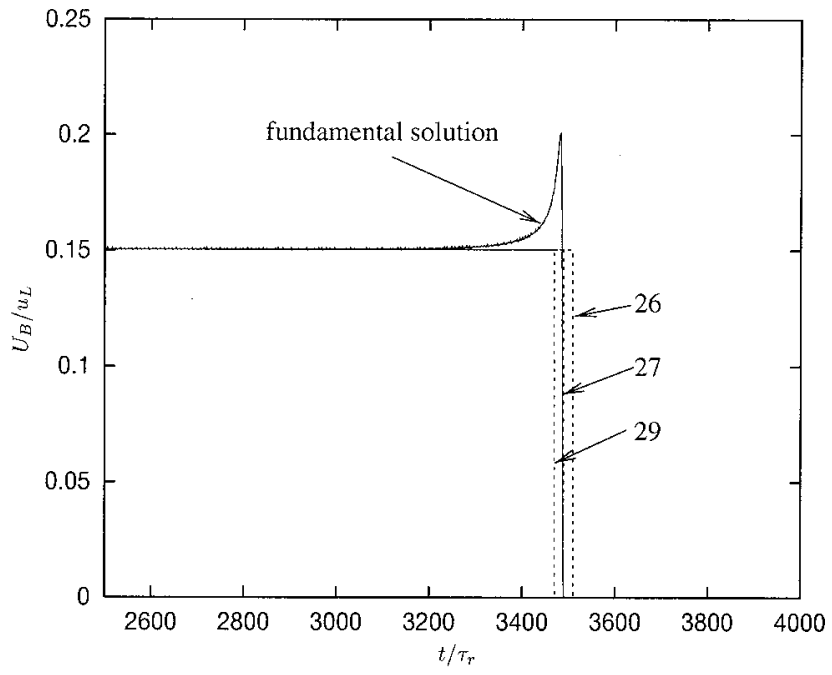

FIG. 9. The burning rate $U_{B}(t) / u_{L}$ against the time $t / \tau_{r}$ for $m$ $=1, Z=20$ from the fundamental (Eulerian) computed solution (solid line) and the results from the flamepoint model (dotted lines) with $\Delta_{L} / \delta_{L}=26$ (right), 27 (center) and 29 (left).

and it can be adjusted until the above criteria is met.

Figure 9 shows the two-flamelet collision process from the fundamental computed solution and from the flamepoint model for $Z=20$, for different $\Delta_{L}$. The imposed laminar flame speed in the flamepoint model, $U_{L}$, is obtained from the fundamental solution. We see that there is indeed a unique value to $\Delta_{L}$ corresponding to when the collision processes from the fundamental solution and from the flamepoint model vanish at the same time. Apart from the fundamental solution (solid line) three cases from the flamepoint model are shown; for $\Delta_{L}=26,27$, and 29 (dotted lines). The results are quite sensitivity to the value of $\Delta_{L}$ taken; only the case with $\Delta_{L}=27$ matches the fundamental solution exactly. For, $m=1$, and $Z=20,40$ and 80, we have obtained, respectively, $\Delta_{L}=27,43$ and 53 .

\section{BURNING RATES OF GROUPS OF FLAMELETS}

A theory for ideal flamelets, $\Delta_{L}=0$

The results of the previous section raises the question of whether it is possible that within some parameter range we can construct a theory for the burning rate by ignoring the interaction time all together, i.e., if we set $T_{i}=0$ ? Let us first consider the ideal flamelet limit which has zero laminar flame thickness $\Delta_{L}=0$.

Consider $N_{0}$ ideal zero-thickness flamelet-pairs released on some set $\left\{x_{n}\right\}$ within the domain of interest at time $t$ $=0$. Two neighboring flamelets moving towards each other with combined speed $2 U_{L}$ consume the enclosed mass between them and vanish instantaneously upon impact $\left(T_{i}\right.$ $=0)$. Thus, a sequence of two-flamelet collisions occurs, and if there are $2 N(t)$ flamelets in the domain at time $t$ then the total burning rate is given approximately by Eq. (10). $N(t) \rightarrow 0$ as $t$ increases. Figure 10 illustrates the situation.

First, we consider an initial algebraic spiral-cut distribution of flamelets, with power exponent $p>0, x_{n}=X_{1} n^{-p}$ for $X_{1}>0$ and $n=1, \ldots, N_{0}$, where $N_{0}=N(t=0) \gg 1$ is the initial number of flamelet-pairs released. The set $\left\{x_{n}\right\}$ has space-filling properties characterized by a box-counting di- 


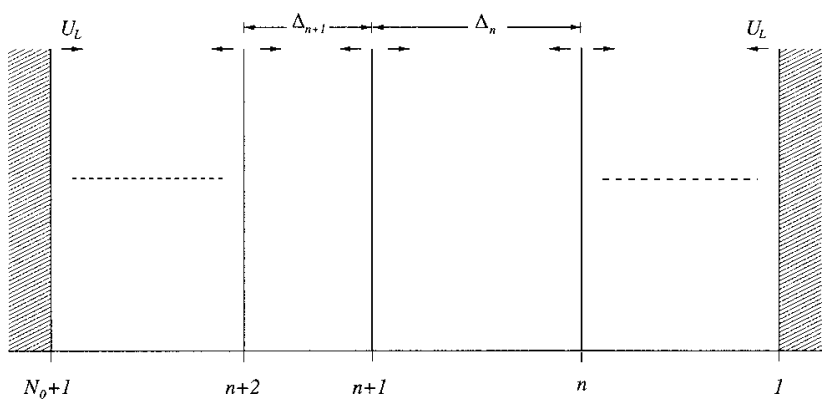

FIG. 10. A sketch of the initial configuration of idealized zerothickness flamelets in the computational domain illustrating the labeling scheme for the algebraic spiral distribution of points.

mension $D_{k}^{\prime}=1 /(1+p)>0$. At time $t=0$, a flamelet-pair is released centered at each location $x_{n}$. Strictly, $U_{L} \rightarrow 0$ as $Z$ $\rightarrow \infty$, so we assume that $Z$ is large but finite so that we have a nonzero flame speed $U_{L}$. The left hand edge of the domain corresponds to the $n=\left(N_{0}+1\right)$ flamelet positioned at $x_{N_{0}+1}$. The right hand edge of the domain correspond to an extra flamelet propagating in to the domain; this does not affect the theoretical results so long as $N \gg 1$.

We label the flamelet pairs according to their initial numbering $n ; n$ is increasing from the right of the domain to the left of the domain, see Fig. 5(a). The initial distance between the $(n+1)^{\text {th }}$ and $n^{\text {th }}$ oncoming flamelets is $\Delta_{n}=p n^{-(p+1)}$ for $n>O(1)$. For $n=N_{0}$, we have $\Delta_{N_{0}}=p N_{0}^{-(p+1)}$, which is the smallest distance between any two flamelets initially; these flamelets collide and vanish at time $T_{0}=\Delta_{N_{0}} / 2 U_{L}$. Up to this time, there is no interaction at all and the total burning rate is exactly

$$
U_{B}(t)=2 U_{L}\left(N_{0}+1\right), \quad 0 \leqslant t<T_{0} .
$$

At $t=T_{0}$, the distance between the next pair of neighboring flamelets is now $\Delta_{N_{0}-1}^{2}=\Delta_{N_{0}-1}-\Delta_{N_{0}}=p(p+1) N_{0}^{-(p+2)}$; and this process continues for successive collisions. Generally, at the time $t_{N}>T_{0}$ of the $n=N$ collision there are $2 N\left(t_{N}\right)$ remaining flamelets in the domain, and the distance between the $n=(N+1)$ and the $n=N$ oncoming flamelets is $\Delta_{N}^{2}\left(t_{N}\right)=p(p+1) N\left(t_{N}\right)^{-(p+2)}$ for $N \gg 1$.

The time lag between the $n=(N+1)$ and $n=N$ collisions is $\Delta t_{N}=t_{N}-t_{N+1}=\Delta_{N}^{2} / 2 U_{L}$.

After each collision the burning rate decreases by $2 U_{L}$ as two flamelets disappear from the domain, i.e., $\Delta U_{B}\left(t_{N}\right)=$ $-2 U_{L}$. Thus, the rate of change in the burning rate between successive collisions is

$$
\begin{gathered}
\frac{\Delta U_{B}\left(t_{N}\right)}{\Delta t_{N}} \sim-2 U_{L} /\left(\frac{N\left(t_{N}\right)^{-(p+2)}}{2 U_{L}}\right), \quad t \geqslant T_{0} \\
\sim-\left(t_{N} / T_{0}\right)^{-(p+2) /(p+1)}, \quad t \geqslant T_{0} \\
\sim-\left(t_{N} / T_{0}\right)^{-\left(1+D_{k}^{\prime}\right), \quad t \geqslant T_{0} .}
\end{gathered}
$$

In the limit that $N \rightarrow \infty$ we have $\Delta t_{N} \rightarrow d t \rightarrow 0$ and $t_{N} \rightarrow t$. Thus,

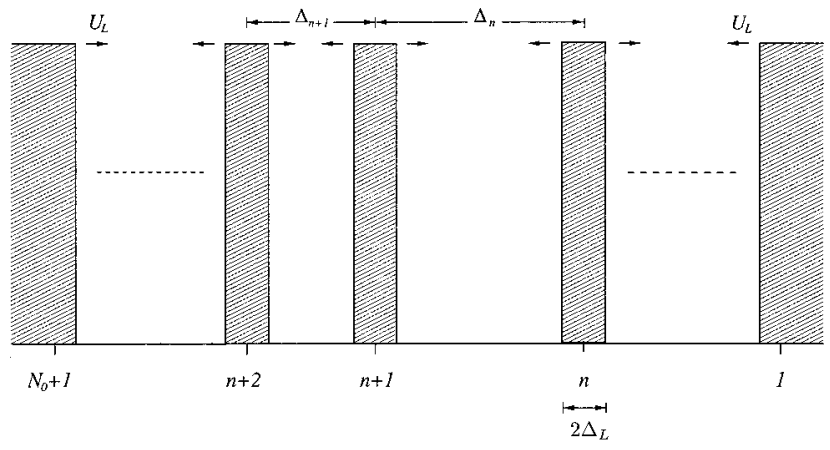

FIG. 11. A sketch of the initial configuration of finite-thickness, $\left(\Delta_{L}>0\right)$, flamelets. Otherwise similar to Fig. 10.

$$
\begin{aligned}
\frac{\Delta U_{B}\left(t_{N}\right)}{\Delta t_{N}} & \rightarrow \frac{d U_{B}(t)}{d t} \sim-\left(t / T_{0}\right)^{-\left(1+D_{k}^{\prime}\right)}, \quad t \geqslant T_{0} \\
\Rightarrow U_{B}(t) & =U_{B}(0)\left(\frac{t}{T_{0}}\right)^{-D_{k}^{\prime}} \\
& =2 U_{L}\left(N_{0}+1\right)\left(\frac{t}{T_{0}}\right)^{-D_{k}^{\prime}}, \quad t \geqslant T_{0} .
\end{aligned}
$$

In (13), we have assumed that the discrete quantity passes over smoothly into the differential quantity and that the latter exists. Note that the relative change in the burning rate upon each collision diminishes to 0 as $N$ increases, viz

$$
\frac{\Delta U_{B}(t)}{U_{B}(t)} \sim \frac{1}{N} \rightarrow 0 \quad \text { as } N \rightarrow \infty .
$$

\section{Algebraic spiral}

For finite thickness flamelets, while still assuming zerointeraction time (Eq. 11), the effective distance that flamelets travel before interacting is reduced to $\Delta_{N}-2 \Delta_{L}$; see Fig. 11. Thus the time till the $n=N$ collision is now

$$
t_{N}=\frac{\Delta_{N}-2 \Delta_{L}}{2 U_{L}} .
$$

The rest of the derivation follows as before, and it is easy to show that the burning rate of a set of finite thickness flamelets with spiral-cut initial configuration is

$$
\begin{gathered}
U_{B}(t) \approx 2 U_{L}\left(N_{0}+1\right), \quad t \leqslant T_{0} \\
U_{B}(t) \approx 2 U_{L}\left(N_{0}+1\right)\left(\frac{t+T_{L}}{T_{0}+T_{L}}\right)^{-D_{k}^{\prime}}, \quad t>T_{0},
\end{gathered}
$$

so long as $N(t) \gg 1 ; \quad T_{0}=\left(\Delta_{N_{0}}-2 \Delta_{L}\right) / 2 U_{L}$, and $T_{L}$ $=\Delta_{L} / U_{L}$.

The range of $Z$ for which the above regime is valid is now examined. The fast interaction hypothesis of Eq. (11) requires that $T_{i} \ll T_{L}$ and also $T_{I} \ll T_{0}$ :

$$
T_{i} \ll \min \left\{\left(\frac{\Delta_{L}}{U_{L}}\right),\left(\frac{\Delta_{N_{0}}}{U_{L}}\right)\right\} .
$$


If we ensure that $\Delta_{N_{0}}>\Delta_{L}$, i.e., that $N(t)$ $<\left(p X_{1} / \Delta_{L}\right)^{1 /(p+1)}$, then the first condition is always dominant. As $Z \rightarrow \infty, U_{L} \rightarrow Z^{-(m+1) / 2} u_{l}$ (Eq. 8); substituted in to (18) this leads to

$$
Z \gg\left(\frac{T_{i} u_{L}}{\Delta_{L}}\right)^{2 /(m+1)} .
$$

For $T_{i}=0$, this simply amounts to $Z>0$.

Furthermore, the flamelet-pairs should not be so closely positioned that successive interactions occur almost on top of each other, (this leads to an explosivelike burning rate which we do not examine in this paper). This restriction means that $\Delta_{N}^{2}>O\left(\Delta_{L}\right)$. leading to

$$
N<\left(\frac{p(p+1) X_{1}}{\Delta_{L}}\right)^{1 /(p+2)} .
$$

More generally, suppose that $\Delta_{L}$ scales with $Z$ like $\Delta_{L}$ $\sim Z^{\alpha}$, then we can re-express this in terms of $Z$ as

$$
Z<O\left(N^{(p+2) / \alpha}\right), \quad \alpha \neq 0 .
$$

If we take $\Delta_{L}$ based on the scaling $\sim D_{\theta} / U_{L}$ then $\Delta_{L}$ $\sim Z^{m+1 / 2}$, with $\alpha=m+1 / 2$, in which case we obtain $Z$ $<O\left(N^{2(p+2) / m+1}\right)$. For $\alpha=0, \Delta_{L}$ attains to a constant value as $Z \rightarrow \infty$, i.e., $\Delta_{L}$ is asymptotically independent of $Z$ in this limit, and therefore there is no upper bound on $Z$; we only require $1 \ll N<\left(X_{1} / \Delta_{L}\right)^{1 /(p+2)}$.

\section{Exponential spiral (Geometric)}

The burning rate of flamelets with an exponential spiralcut distribution, $\left\{x_{n}\right\}$, in the initial condition can be derived in a similar manner. $\left\{x_{n}\right\}$ is in fact a geometric progression, viz $x_{n}=R^{n} x_{0}$ for $n=0, \ldots, N_{0}$, with the geometric ratio $R$ $=e^{a}$ (Sec. III). The anomalous burning rate for finite thickness flamelets is

$$
\begin{gathered}
U_{B}(t)=2 U_{L}\left(N_{0}+1\right), \quad 0 \leqslant t<T_{0} \\
=2 U_{L}\left(N_{0}+1\right)+2 U_{L} \ln \left(\frac{t+T_{L}}{T_{0}+T_{L}}\right)^{-1 /(R-1)}, \quad t \geqslant T_{0} .
\end{gathered}
$$

This is valid for $N \gg 1$, and for $T_{i} \ll \Delta_{L} / U_{L}$. The latter gives the same lower bound on $Z$ as in Eq. (19). The restriction $\Delta_{L}^{2}>O\left(\Delta_{L}\right)$ gives $(R-1)^{2} x_{0}>\Delta_{L}$. If we again assume that $\Delta_{L} \sim Z^{\alpha}$, then this requires $Z<\left((R-1)^{2} x_{0}\right)^{1 / \alpha}$ for $\alpha \neq 0$. For $\alpha=0$, there is no restriction on $Z$. There is also no upper bound on $N$ since in this geometric case the distances between flamelets is increasing with $n$, see Fig. 5(b).

Note that, apart from the logarithm, the overall form of Eqs. (22) and (23) is similar to Eqs. (16) and (17), especially the nondimensional time variable in the argument of $\ln (\cdot)$, and the appearance of the geometric ratio $R$ in the exponent of the time variable.

\section{Fractal Cantor Dust}

We consider the middle third Cantor fractal dust which is the simplest type of homogeneous fractal. It can be readily constructed using a simple recurrence relation as follows [Fig. 5(c)]: (1) let $E_{0}$ be the unit interval [0,1]. (2) Let $E_{1}$ be the set obtained by deleting the middle third of the line interval, so that $E_{1}=[0,1 / 3] \cup[2 / 3,1]$. Now remove the middle third from each of these two subintervals to obtain $E_{2}=\left[0,1 / 3^{2}\right] \cup\left[2 / 3^{2}, 1 / 3\right] \cup\left[2 / 3,7 / 3^{2}\right] \cup\left[8 / 3^{2}, 1\right]$. Continue this procedure, $E_{k}$ being obtained from $E_{k-1}$ by removal of all middle third's of the subintervals. $E_{k}$ consists of $2^{k}$ intervals of length $(1 / 3)^{k}$.

In general, for simple fractals such as the Cantor Dust, the fractal dimension $H$ is equal to its similarity dimension $S$ which can be readily obtained by the following rule: if a set is made up of $M$ copies of itself when scaled by a factor $r$, then $S=\ln (M) / \ln (1 / r)$. For the middle third Cantor Dust, we have a scaling factor of $r=1 / 3$ which produces $M=2$ copies of itself, thus $H=S=\ln (2) / \ln (3)=0.631$. There are generalizations to the Cantor Dust with $0<H<1$, so in the remainder of this paper we will refer to the general case. The Cantor Dust scales according to the Hausdorff measure such that the number of boxes of size $\epsilon$ needed to cover the fractal set is given by

$$
N(\epsilon)=\left(\frac{X}{\epsilon}\right)^{H}
$$

where $X$ is the extent of the domain which contains the fractal set.

In practice, there is some uppermost generation $J$ so that the smallest line segment has a length $\epsilon_{J}=X r^{J}$. Consider a set of flamelet-pairs of finite thickness $\Delta_{L}$ initiated on the nodes of a fractal Cantor Dust of fractal dimension $H$ with maximum generation $J>0$. The first interaction occurs at $T_{0}=\left(\epsilon_{J}-2 \Delta_{L}\right) / 2 U_{L}$. Thus, with $X=1$,

$$
U_{B}^{J}(t)=2 U_{L} N\left(\epsilon_{J}\right)=2 U_{L} r^{-J H}, \quad 0 \leqslant t<T_{0} .
$$

Subsequent collisions occur at $t_{j}=\left(\epsilon_{j}-2 \Delta_{L}\right) / 2 U_{L} \approx r^{j} / 2 U_{L}$ for $\epsilon_{j} \gg 2 \Delta_{L}$; the time lag between successive collisions is $\Delta t_{j}=t_{j-1}-t_{j} \sim\left(r^{-1}-1\right) t_{j}$. The change in the burning rate between collisions is given by the fractal measure at generation $j$, viz

$$
\Delta U_{B}^{j}\left(t_{j}\right)=2 U_{L}\left(N_{j-1}-N_{j}\right) \approx-2 U_{L}\left(1-r^{H}\right)\left(t_{j}\right)^{-H} .
$$

We observe that whereas in the algebraic-spiral case, where $\Delta U_{B} / U_{B} \rightarrow 0$ as $N \rightarrow \infty$ (Eq. 14), in the fractal case we have

$$
\frac{\Delta U_{B}^{j}}{U_{B}^{j}}=-\left(1-r^{H}\right)=- \text { const }<0
$$

for any $j$. Thus, the relative change in the burning rate at each collisions does not converge to zero and this implies that the limit 


$$
\lim _{j \rightarrow \infty} \frac{\Delta U_{B}^{j}}{\Delta t_{j}} \rightarrow \frac{d U_{B}(t)}{d t} \sim t^{-(1+H)}, \quad j \gg 1
$$

does not exist. Consequently, it follows that the burning proceeds in discrete steps such that within any time-generation the burning rate is constant,

$$
U_{B}^{j}(t)=2 U_{L} r^{-j H}, \quad t_{j} \leqslant t<t_{j-1} .
$$

where $t_{j}=\left(\epsilon_{j}-2 \Delta_{L}\right) / 2 U_{L}$.

In fact, if the continuous limit is taken in Eq. (18), the solution represents the envelope (upper-bound) of the burning rate;

$$
U_{B}^{u}(t)=U_{B}(0)\left(\frac{t+T_{L}}{T_{0}+T_{L}}\right)^{-H} \quad j \gg 1, \quad t>T_{0} .
$$

The parameter range for which Eq. (30) is valid can be obtained from similar considerations as that for the algebraic-spiral case. The requirement that $T_{i} / T_{L} \ll 1$ leads to the same lower bound on $Z$ as in Eq. (19). We also require $\epsilon_{J} \gg \Delta_{L}$, which leads to an upper bound on the number of generations $J$,

$$
J \ll \ln \left(\frac{\Delta_{L}}{X}\right) / \ln (r) .
$$

In terms of $Z$, if we assume that $\Delta_{L} \sim Z^{\alpha}$, then this gives

$$
Z \ll O\left(r^{J / \alpha}\right), \quad \alpha \neq 0
$$

If we take $\alpha=0$, then there is no upper bound on $Z$.

It is appropriate here to note that although the underlying geometries in the spiral cases and the fractal case are different, the form of the anomalous burning regimes (or its envelope) are strikingly similar; compare Eqs. (17), (23), and (30).

\section{THE FLAMEPOINT MODEL}

\section{A. Description}

Computing the fundamental solutions for the fractal geometries presents severe problems. Even taking just $J=10$ generations means that the range of scales is $3^{10} \approx 10^{5}$ which must be resolved at the smallest scale $\Delta_{L}$. This is clearly beyond simple techniques. We have therefore developed a Lagrangian model for flamelet propagation to compute results for the fractal case.

The assumption of instantaneous flamelets collision and mass consumption, Eq. (11), leads to the idea of modeling flamelet propagation as a thin point, or slab, of thickness $\Delta_{L}$ which propagates with speed $U_{L}$ in a Lagrangian frame of reference along the real line. Upon collision, two linesegments (flamelets) are consumed immediately and vanish from the domain. In this flamepoint model, a finite thickness is given to the flamelet by the rule that when two linesegments (flamelets) come within a distance of $2 \Delta_{L}$ of each other they are designated as consumed and they vanish from the domain. The flame speed $U_{L}$ is imposed as the motion of

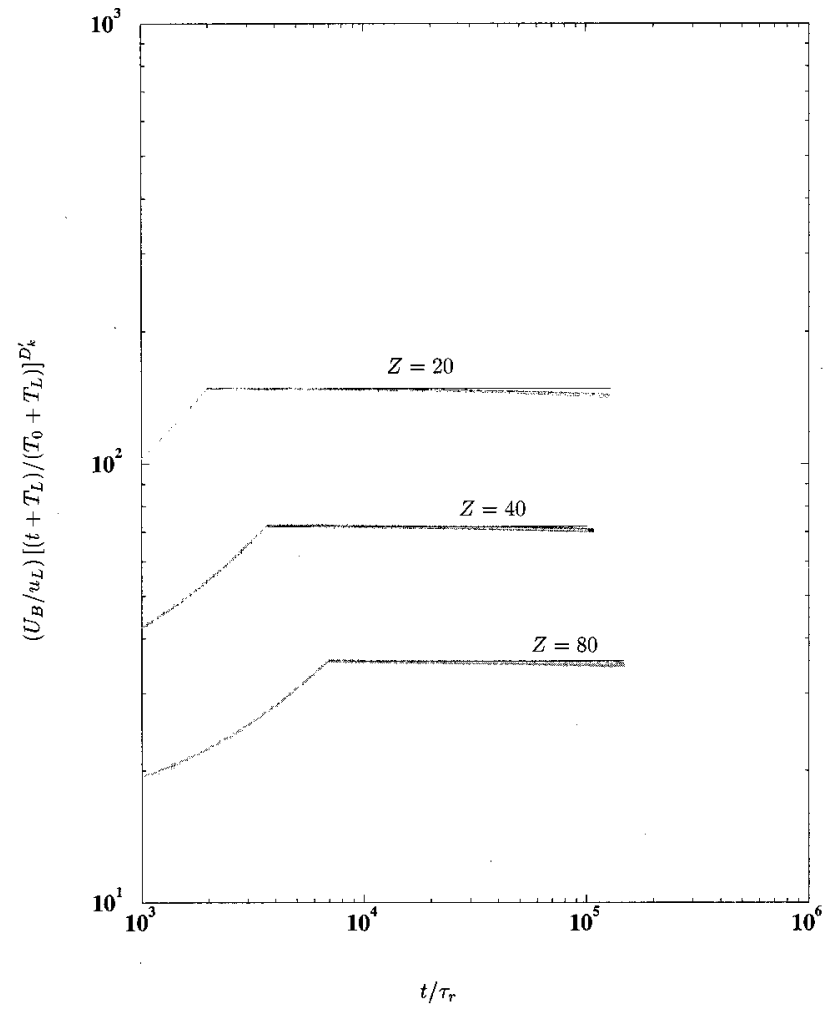

FIG. 12. Log-log plots of the normalized burning rates $\left(U_{B} / u_{L}\right)\left(t+T_{L} / T_{0}+T_{L}\right)^{D_{k}^{\prime}}$ against the time $t / \tau_{r}$, for flamelets released in an algebraic-spiral field with $p=0.5$, and thus $D_{k}^{\prime}=1 /(1$ $+p)=2 / 3$. Three results with $m=1, Z=20,40$, and 80 are shown. The solid lines are the theoretical curves of Eqs. (16) and (17); the dashed lines are the computed solutions of Eq. (4); the dot-dashed lines are the results from the flamepoint model described in Sec. VI. (These last two results coincide closely and can hardly be distinguished by eye.)

the flamepoint, it is not an output. Numerically, all we need to do is to keep track of the number of flamelets in the domain at time $t$. This number is reduced by two every time two flamepoints collide.

\section{B. Validation}

To validate the flamepoint model, results from this model must be compared to those from the fundamental solutions; we will have to anticipate the results of the latter which are described in the next section. Results from the flamepoint model and the fundamental solutions, for the same effective parameter values in spiral fields, are plotted in Figs. 12 and 13. The flamepoint model results match the physical solutions exactly - it is difficult to distinguish the two sets of results by eye. This adequately validates the flamepoint model.

The usefulness of the Lagrangian based flamepoint model is that it is extremely fast; whereas solving the equations of motion even in one-dimensions takes up to $24 \mathrm{hrs}$ on supercomputers for the cases described above, the flamepoint model takes minutes on a workstation to generate the same results. This is important for two reasons. First, it allows us to examine parameter ranges which are inaccessible to the fundamental model in one-dimensions. Secondly, it opens up the possibility of performing these kinds of simulations in 


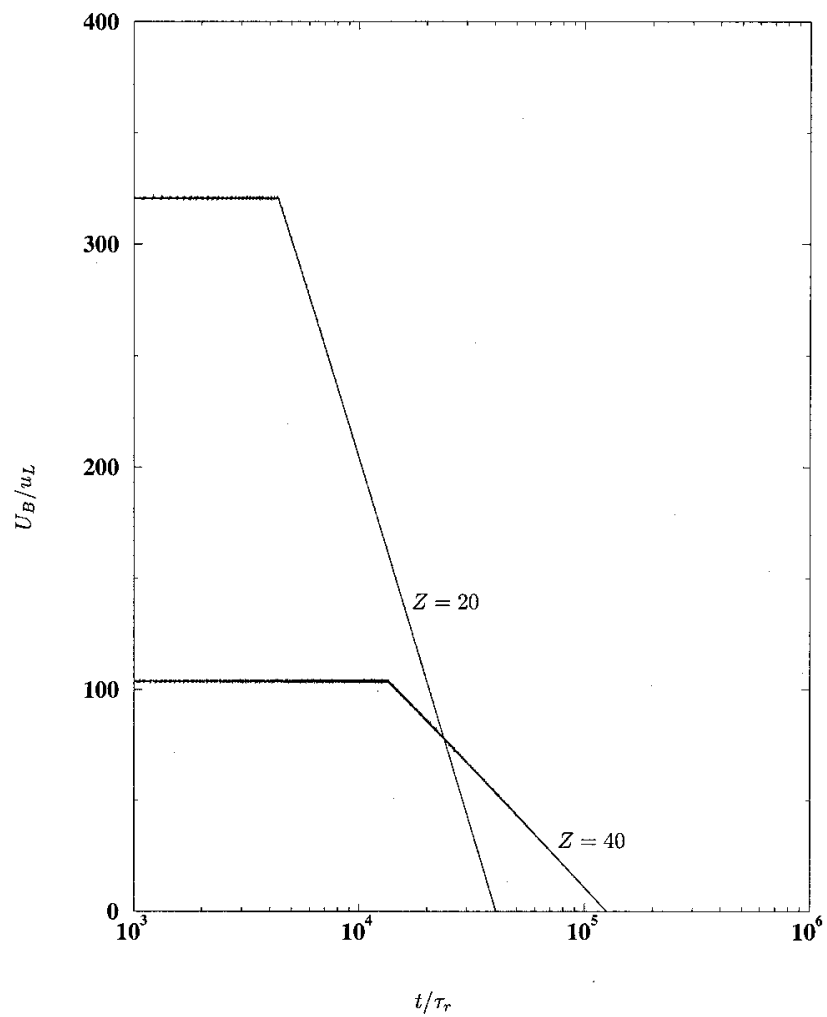

FIG. 13. Log-linear plots of the burning rates $\left(U_{B} / u_{L}\right)$ against the time $t / \tau_{r}$, for flamelets released in a exponential spiral-cut field. Results for two cases are shown, $Z=20, R=1.001$ and $Z=40, R$ $=1.0015$. The solid lines are the theoretical curves of Eqs. (22) and (23); the dashed lines are the computed solutions for Eq. (4); the dot-dashed lines are the results from the flamepoint model described in Sec. VI. (All three cases coincide closely and can hardly be distinguished by eye.)

two- and three-dimensions. (However, in more than onedimensions one would have to exercise caution since the interaction of flames is now curvature dependent. Furthermore, flame propagation at the formation of cusps is not well understood yet. Within a Lagrangian framework, these effects would have to be modeled even if other physical effects are ignored.)

\section{RESULTS}

The nonlinear reaction-diffusion equation (4) with reaction rate equation (7) was solved numerically for different values of the Zeldovich number $Z$, for $m=1$. Flamelet-pairs were released with initial spiral-cut distributions as discussed in Sec. III. A Crank-Nicholson finite difference scheme was found to be adequate. A high density of grid points is necessary to resolve the thin flamelet structure and the reaction zone; typically, we used a density of $2^{23}$ grid points per unit length.

$\theta(x, t)$ evolves in time inside the domain of interest; the mass flux $M(t)$ is the computed area under the $\theta$ distribution within this domain as given in Eq. (5). This area is computed by Simpson's rule. The burning rate is given by Eq. (6) which we compute from $M(t)$ as a second order finite difference.

\section{A. Algebraic spiral distribution}

The burning rates of flamelets in an algebraic spiral-cut field was computed for $m=1, p=0.5$ which gives $D_{k}^{\prime}$ $=1 /(p+1)=2 / 3$. Figure 12 shows the results for three cases, $Z=20,40$ and 80 (dotted lines). The figure shows the log$\log$ plots of $\left(\left(t+T_{L}\right) /\left(T_{0}+T_{L}\right)\right)^{D_{k}^{\prime}}\left(U_{B} / u_{L}\right)$ against $t / \tau_{r}$. Also shown on this figure are the corresponding theoretical curves, Eqs. (16) and (17) (solid lines); these theoretical curves overlap the computed physical solution when $N(t)$ $\gg 1$. As $N(t)$ decreases, the theoretical curves deviate from the physical solutions at later times, which is clearly seen for $Z=20$.

\section{B. Exponential spiral (geometric) distribution}

The burning rates of flamelets in an exponential spiral-cut field of flamelets was computed for two cases, $R=1.001$ and $R=1.0015$. The results are shown in Fig. 13 as log-linear plots of $U_{B}(t) / u_{L}$ against the time $t / \tau_{r}$. The corresponding theoretical curves, Eqs. (22) and (23), are also shown on the same plots. Again we see that the overlap with the theoretical curves is nearly exact. In these particular cases, the theoretical solution remains valid for almost the entire duration of the burning process, until $N(t)=O(10)$. This reflects the fact that the exponential spiral is a milder singularity than the algebraic spiral.

\section{Fractal geometry}

In view of the excellent agreement of the flamepoint model with the computed results for the spiral fields, we can use the flamepoint model to compute the results for the fractal field where the finite difference method needs excessively large computational time.

Figure 14 shows the log-log plot of the burning rate $U_{B}(t) / u_{L}$ against time $t / \tau_{r}$ obtained from the flamepoint model. We can take $X=1, D_{\theta}=1$, and $\tau_{r}=1$, which gives $u_{L}=1$ and $\delta_{L}=1$. The input to the flamepoint model are $U_{L}=1$, and $\Delta_{L}=0.1 \epsilon_{J}$, where we have taken $J=9$ and so $\epsilon_{J}=(1 / 3)^{9}$. (Thus, the scale separation between the smallest and largest scales is $1 / \epsilon_{J}=3^{9}$.) We observe a stepwise discontinuous burning process, with a drop of a factor of $1 / 2$ in the burning rate at every collision step, so long as $j>4$, as predicted in Eqs. (27) and (29). Also shown on Fig. 14 is the theoretical curve for the envelope to the burning rate $U_{B}^{u}(t)$, Eq. (30).

\section{SUMMARY AND COMMENTS}

We have examined the burning regimes of groups of flamelets in self-similar multiple scale fractal and nonfractal fields. We have found that these fields induce anomalous rates of burning which are determined by the space-filling character of the initial field, whether this is defined by the capacity $D_{k}$, the fractal dimension $H$, or the geometric ratio $R$.

We have contrasted three different types of geometries with distinct space-filling characteristics. (In the following we refer to $D_{k}$ rather than $D_{k}^{\prime}$ since the context makes it clear that we are referring to the one-dimensional line-cut in the spiral cases): (1) The algebraic spiral which has fractal di- 


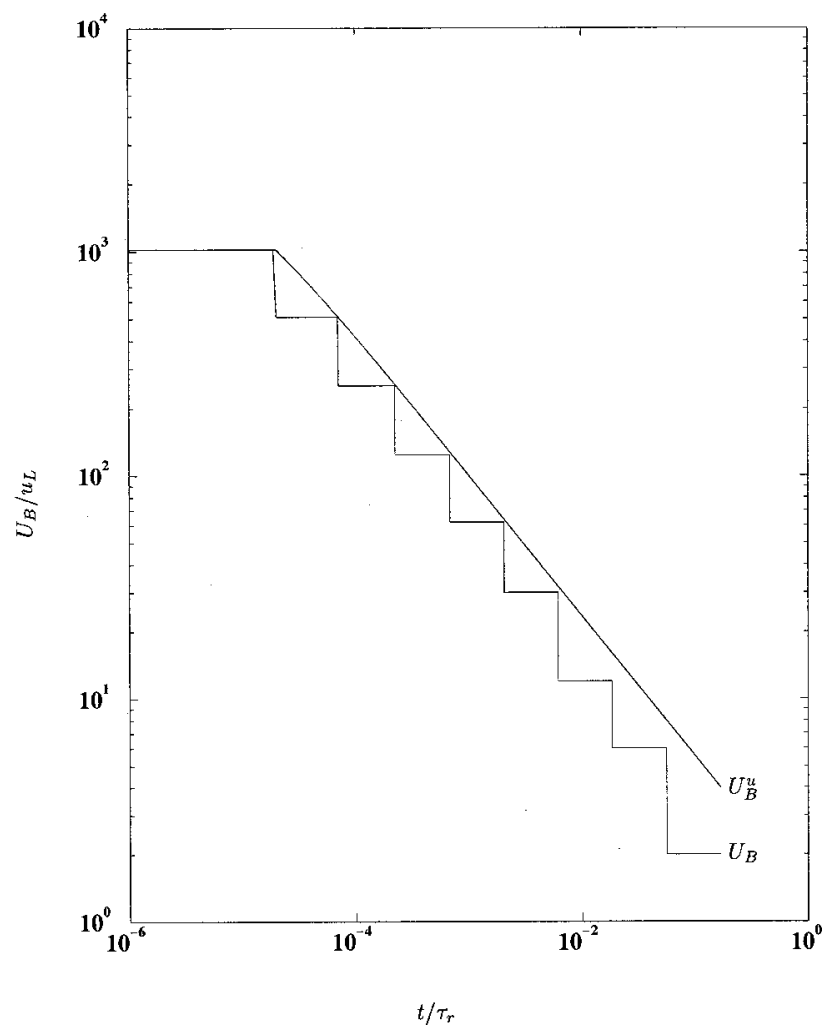

FIG. 14. Log-log plot of the burning rate $\left(U_{B} / u_{L}\right)$ against the time $t / \tau_{r}$, for flamelets released in a fractal Cantor Dust field. $U_{B}$ is the result from the flamepoint model. $U_{B}^{u}(t) / u_{L}$ is the theoretical solution for the envelope in Eq. (30).

mension $H=0$, and capacity $D_{k}>0$; (2) the exponential spiral which has $H=0, D_{k}=0$ and geometric ratio $R>1$; (3) the fractal Cantor dust which has $H=D_{k}>0$. Although the specific burning regimes induced by the different geometries are different in each case [Eqs. (16), (17), (22), (23), (29), and (30)], all of these results can, however, be brought in to a universal form, viz

$$
U_{B}(t) \sim \mathcal{F}\left(\tau^{-\zeta}\right)
$$

where $\mathcal{F}$ is a function determined by the initial field; $\tau=t$ $+T_{L} / T_{0}+T_{L}$ is nondimensional time variable; $\zeta$ is the exponent which contains the space-filling 'signature' which is also determined by the initial field. $\mathcal{F}$ and $\zeta$ take the following forms in the three cases considered:

(1) Algebraic spiral: $\mathcal{F}(\cdot) \equiv 1(\cdot)$, and $\zeta=D_{k} ; \mathcal{F}$ is continuous.

(2) Exponential spiral: $\mathcal{F}(\cdot) \equiv \ln (\cdot)$, and $\zeta=1 /(R-1) ; \mathcal{F}$ is continuous.

(3) Fractal Cantor dust: $\mathcal{F}^{u}(\cdot) \equiv 1(\cdot)$, and $\zeta=H$ for the envelope (upper-bound). $\mathcal{F}$ itself is stepwise discontinuous.

We note that as the fields become more and more sparse $\left(D_{k} \rightarrow 0\right.$, or $H \rightarrow 1$, or $\left.R \rightarrow \infty\right)$ then $U_{B} \rightarrow$ Const. This is interpreted as the case of an infinite domain in which the flamelets are arranged far apart from each other so that there is never any interaction, hence the burning rate is simply the sum of individual flamelets. When the algebraic spiral field or the fractal field become space-filling, $D_{K} \rightarrow 1$ or $H \rightarrow 1$, then $\zeta \rightarrow 1$, and hence $U_{B} \rightarrow \tau^{-1}$. For the algebraic spiral field, $p \rightarrow 0$ as $D_{k} \rightarrow 1$; and in view of the restriction in Eq. (20), this requires that $X_{1} \sim 1 / p$ as $p \rightarrow 0$. As the exponential spiral field becomes space-filling, $R \rightarrow 1$, then $\zeta \rightarrow \infty$. We require $x_{0} \sim 1 /(R-1)^{2}$.

The exponential spiral has not been considered in previous works, and the results here show that it is not just fractallike fields (in the sense that either $D_{K}>0$ or $H>0$ ) which can induce anomalous physical regimes; self-similar multiple scale geometry appears to be enough. However, it is interesting that the exponential spiral induces a log-regime while the other two fractal-like geometries induce a power law in $\tau$. This reflects the milder singularity of the exponential spiral.

The similarity of the burning regimes induced by the algebraic spiral and the fractal fields is striking. The underlying generator for the fractal field is a fixed scaling ratio $r$ (Sec. V 3) and yet it induces a burning regime whose average behavior is identical to the regime induced by the algebraic spiral field rather than the exponential spiral field; compare (17) and (30). However, the actual burning process in a fractal field occurs in stepwise fashion, Fig. 14.

The flamepoint model was developed based on the idea of representing flamelet propagation in a Lagrangian frame of reference as a point with finite thickness $\Delta_{L} . \Delta_{L}$ was defined with respect to the two-flamelet collision process (in Sec. VI) as a type of flamelet interaction length scale, and it can be determined uniquely. This makes the flamepoint model physically appealing, which is an advantage over many Lagrangian based models where ad hoc assumptions for the flame thickness must be made. This leads to an accurate model for flamelet propagation; the results of the flamepoint match those of the fundamental solutions of the PDE (4) almost exactly for the two spiral cases, Figs. 12 and 13. The flamepoint model was then used to obtain results for the fractal Cantor dust case, Fig. 14.

Finally, we may contrast the anomalous decaying regimes in Burgers turbulence [1] for one-signed pulses, to the anomalous burning regimes which takes the general form of equation (33) with $\zeta=\left\{D_{k}, H\right\}$. Even though they are different physical systems, the functional form of the energy decay law in the advection-diffusion system (see Eq. 3.15 in [1]) is similar to (33); however, the dependence on the capacity is different, viz $\zeta=(1-D) /(2-D)$.

In the simple linear diffusive decay in [3], the functional form for the decay of scalar variance, $S(t, D)$, is similar to (33) but with $\zeta=(D-1) / 2$ for fractal or spiral fields; see Eq. (8) of [3]. But when normalized by the classical case for a single pulse $(D=0)$, we obtain $S(t ; D) / S(t ; 0) \sim t^{-D}$. This is the same dependence obtained for the nondimensionalized burning rate of flamelets, (Eqs. (13) and (17) with $S(t ; D)$ $\equiv U_{B}\left(t ; D_{k}\right)$ and $S(t ; 0) \equiv U_{B}\left(t ; D_{k}=0\right)=$ const $)$, which is described by a system of nonlinear reaction-diffusion equations.

Thus, while it appears that the functional form of the anomalous physical regimes takes the general form of Eq. (33), the exact dependence of the power $\zeta$ on the initial field geometry (characterized by its capacity, $D$, or its fractal dimension, $H$, or by the geometric ratio $R$ ), depends of the specific physical regime of interest. Nevertheless, it has been shown here that the nondimensionalized burning rate in the nonlinear reaction-diffusion flamelet regime, is similar to the 
nondimensionalized scalar decay rate in the linear diffusion regime, with $\zeta=D$. (Physical quantities here are nondimensionalized by the same quantities for the classical case when $D=0$.) This last result is particularly interesting since the process of scalar diffusion, collision and merging, which leaves behind a single pulse with a larger correlation length than the originals, is so different to the propagation and collision of flamelets which happens almost instantaneously and

[1] S. N. Gurbatov and D. G. Crighton, Chaos 5, 524 (1995).

[2] J. R. Angilella and J. C. Vassilicos, Physica D 124, 23 (1998).

[3] J. C. Vassilicos, Phys. Rev. E 52, R5753 (1995).

[4] M. V. Berry, Physica D 38, 29 (1989).

[5] Z.-S. She, E. Aurell, and U. Frisch, Commun. Math. 148, 623 (1992).

[6] M. van den Berg, Probab. Theory Related Fields 100, 439 (1994). two flamelets "disappear" from the domain upon collision as the burning process is completed.

\section{ACKNOWLEDGMENTS}

The authors gratefully acknowledge financial support from the Hong Kong Research Grant Council. Computing resources were made available at the Hong Kong University of Science and Technology.

[7] J. Fleckinger, M. Levitin, and D. Vassiliev, Proc. London Math. Soc. 71, 372 (1995).

[8] I. Stewart, New Sci. 156, 22 (1997); R. Lewin, New Sci. 162, 34 (1999).

[9] F. A. Williams, Combustion Theory, 2nd ed. (Benjamin/ Cummings Pub., Menlo Pk., California, 1985).

[10] P. Clavin, Prog. Energy Combust. Sci. 11, 1 (1985).

[11] K. Falconer, Fractal Geometry. Mathematical Foundations and Applications (John Wiley, Chichester, 1990). 